\title{
WATER WAVES GENERATED BY DISTURBANCES AT AN ICE COVER
}

\author{
PARAMITA MAITI AND B. N. MANDAL
}

Received 13 June 2004 and in revised form 30 November 2004

This paper is concerned with two-dimensional unsteady motion of water waves generated by an initial disturbance created at an ice sheet covering the water. The ice cover is modeled as a thin elastic plate. Using linear theory, the problem is formulated as an initial value problem for the velocity potential describing the motion in the liquid. In the mathematical analysis, the Laplace and Fourier transform techniques have been utilized to obtain the depression of the ice-covered surface in the form of an infinite integral. For the special case of initial disturbance concentrated at the origin, taken on the ice cover, this integral is evaluated asymptotically by the method of a stationary phase for a long time and large distance from the origin. The form of the ice-covered surface is graphically depicted for two types of initial disturbances.

\section{Introduction}

The two-dimensional problems concerning generation of water waves due to a prescribed initial displacement or impulse mostly concentrated at a point were discussed in treaties of Lamb [4] and Stoker [6] within the framework of the linearized theory of water waves. Kranzer and Keller [3] considered axially symmetrical initial surface disturbance in water of finite depth and compared the theory with experimental results. Chaudhury [1] extended these results for any initial surface impulse and elevation across arbitrary regions.

All the problems considered by Lamb [4], Kranzer and Keller [3], and Chaudhury [1] involve an ocean with free surface. However, in polar regions, the ocean is generally covered by ice. Two types of models for the ice cover are usually assumed. In the first model, the ice cover is assumed to consist of a thin but uniform distribution of noninteracting materials with no elastic property, known as an inertial surface (e.g., broken ice). Mandal [5] considered generation of water waves due to initial disturbances at such an inertial surface. In the second model, the ice cover is assumed to consist of a thin ice sheet of small thickness $h$, say, of which still a smaller part is immersed in water, the ice sheet being composed of materials having elastic properties. In this paper, we consider the problem of generation of water waves in an ocean of infinite depth covered by such 
an ice sheet due to initial disturbance on the ice cover, the disturbance being taken as an initial depression of the ice cover or an initial impulse on it.

Assuming the linear theory, the problem is formulated as an initial value problem in terms of nondimensional coordinates and nondimensional time. Using the technique of Laplace transform, the problem is reduced to a boundary value problem which is solved by using Fourier transform. The Laplace-Fourier transform of the ice cover depression is then found. After invoking the inverse transforms, the nondimensional form of the ice cover depression is obtained in terms of an integral. In the absence of the ice cover, this reduces to the classical result. This integral is evaluated for large distance and long time for the case when the initial disturbance is concentrated at the origin. The asymptotic form of the depression is displayed graphically for various values of the ice cover parameter, in a number of figures, and compared with the case when there is no ice cover.

\section{Mathematical formulation}

We consider water as an inviscid, incompressible, homogeneous liquid of volume density $\bar{\rho}$. A rectangular cartesian coordinate system $(\bar{x}, \bar{y})$ is chosen in which the $\bar{y}$-axis is chosen vertically downwards into the water and the plane $\bar{y}=0$ coincides with the rest position of the ice cover. The ice cover is modeled as a thin elastic sheet having a uniform surface density $\bar{\epsilon} \bar{\rho}$, where $\bar{\epsilon}$ is a constant having the dimension of length. The motion of the water is created by a sudden initial depression of the ice cover or an impulse on the ice cover. Since the motion starts from rest, it is irrotational and can be described by a velocity potential $\bar{\phi}$, which satisfies Laplace's equation

$$
\nabla_{(\bar{x}, \bar{y})}^{2} \bar{\phi}=0 \text { in the fluid region, }
$$

and the bottom condition

$$
\nabla \bar{\phi} \longrightarrow 0 \quad \text { as } \bar{y} \longrightarrow \infty
$$

The assumption of no cavitation between the ice and water at any time produces the kinematic condition

$$
\bar{\phi}_{\bar{y}}=\bar{\eta}_{\bar{t}} \quad \text { on } \bar{y}=0 \text {, }
$$

where $\bar{\eta}$ is the depression of the ice cover below its mean position, assumed small, and $\bar{t}$ denotes the time.

The linearized dynamic condition at the ice cover is (cf. Chung and Fox [2])

$$
\left(\bar{\phi}-\bar{\epsilon} \bar{\phi}_{\bar{y}}\right)_{\bar{t} \bar{t}}=\left\{1+\bar{D} \frac{\partial^{4}}{\partial \bar{x}^{4}}\right\} g \bar{\eta}_{\bar{t}} \quad \text { on } \bar{y}=0,
$$

where $\bar{D}$ is the flexural rigidity of the ice cover and is given by $\bar{D}=E h^{3} / 12\left(1-v^{2}\right) \bar{\rho} g, E$ being Young's modulus and $\nu$ being Poisson's ratio. 
The initial conditions at the ice cover are

$$
\begin{gathered}
\bar{\phi}-\bar{\epsilon} \bar{\phi}_{y}=0 \quad \text { on } \bar{y}=0, \bar{t}=0, \\
\left(\bar{\phi}-\bar{\epsilon} \bar{\phi}_{\bar{y}}\right)_{\bar{t}}=\left\{1+\bar{D} \frac{\partial^{4}}{\partial \bar{x}^{4}}\right\} \bar{\eta}_{0}(\bar{x}) \quad \text { at } \bar{t}=0, \bar{y}=0,
\end{gathered}
$$

where $\bar{\eta}_{0}(\bar{x})$ is the initial depression of the ice cover.

It is convenient to write the system in a nondimensional form by introducing a characteristic length $l$ and the characteristic time $\sqrt{l / g}$, where $g$ is the gravity. Defining the dimensionless quantities (without the overbar) as

$$
\begin{aligned}
& (x, y)=\frac{(\bar{x}, \bar{y})}{l}, \quad t=\bar{t} \sqrt{\frac{g}{l}}, \quad \eta=\frac{\bar{\eta}}{l}, \\
& \phi=\frac{\bar{\phi}}{l \sqrt{g l}}, \quad D=\frac{\bar{D}}{l^{4}}, \quad \epsilon=\frac{\bar{\epsilon}}{l},
\end{aligned}
$$

we find that $\phi$ satisfies

$$
\begin{gathered}
\nabla^{2} \phi=0 \quad \text { in the fluid region, } \\
\left(\phi-\epsilon \phi_{y}\right)_{t t}=\left\{1+D \frac{\partial^{4}}{\partial x^{4}}\right\} \phi_{y} \quad \text { on } y=0, \\
\nabla \phi \longrightarrow 0 \quad \text { as } y \longrightarrow \infty, \\
\phi_{y}=\eta_{t} \quad \text { on } y=0, \\
\phi-\epsilon \phi_{y}=0 \quad \text { at } t=0, y=0, \\
\left(\phi-\epsilon \phi_{y}\right)_{t}=\left\{1+D \frac{\partial^{4}}{\partial x^{4}}\right\} \eta_{0}(x) \quad \text { at } t=0, y=0,
\end{gathered}
$$

where $\eta_{0}(x)$ is the initial nondimensional depression of the ice cover.

\section{Solution for $\phi$}

Defining joint Laplace-Fourier transform of $\phi(x, y, t)$ as

$$
\widehat{\Phi}(k, y, s)=\frac{1}{(2 \pi)^{1 / 2}} \int_{-\infty}^{\infty} e^{-i k x} \int_{0}^{\infty} e^{-s t} \phi(x, y, t) d t d x,
$$

it is easy to see that $\widehat{\Phi}$ satisfies

$$
\begin{gathered}
\hat{\Phi}_{y y}-k^{2} \hat{\Phi}=0 \quad y \geq 0, \\
\hat{\Phi}_{y}=s \hat{\bar{\eta}}-\hat{\eta}_{0}(k) \quad \text { on } y=0, \\
s^{2} \widehat{\Phi}-\left(\epsilon s^{2}+1+D k^{4}\right) \hat{\Phi}_{y}=\left(1+D k^{4}\right) \widehat{\eta_{0}}(k), \\
\hat{\Phi}_{y} \longrightarrow 0 \quad \text { as } y \longrightarrow \infty .
\end{gathered}
$$

The general solution of (3.2) satisfying (3.5) is

$$
\widehat{\Phi}=A(k, s) e^{-k|y|},
$$


where the unknown function $A(k, s)$ is obtained by using (3.4). We are interested in the depression $\eta(x, t)$ of the ice cover. Its Laplace-Fourier transform $\hat{\bar{\eta}}$ is obtained by using (3.3) and is given by

$$
\hat{\bar{\eta}}=\frac{s \hat{\eta}_{0}(k)}{s^{2}+c^{2}}
$$

where

$$
c^{2}=\frac{|k|\left(1+D k^{4}\right)}{(1+\epsilon|k|)} .
$$

If the initial depression of the ice cover is concentrated at the origin, then we can choose $\eta_{0}(x)=\delta(x)$, where $\delta(x)$ is the Dirac delta function and in this case, we find

$$
\hat{\bar{\eta}}=\frac{1}{\sqrt{2 \pi}} \frac{s}{s^{2}+c^{2}} .
$$

After taking inversions, we find that the depression of the ice cover, in this case, is obtained in the form

$\eta(x, t)=\frac{1}{4 \pi}\left[\int_{0}^{\infty} e^{i t(c-k(x / t))} d k+\int_{0}^{\infty} e^{i t(c+k(x / t))} d k+\int_{0}^{\infty} e^{-i t(c-k(x / t))} d k+\int_{0}^{\infty} e^{-i t(c+k(x / t))} d k\right]$.

In the next section, we evaluate $\eta$ for large $x$ and $t$ asymptotically by using the method of stationary phase.

\section{Asymptotic form of $\eta(x, t)$}

We are interested in the waves created by the initial disturbance after a long lapse of time and a large distance from the origin. We use the method of stationary phase to evaluate the integral in (3.10) for large $x$ and $t$ such that $x / t$ remains finite. The second and fourth integrals have no stationary point in the range of integration. Thus these do not contribute to $\eta(x, t)$ for large values of $x$ and $t$.

From the first and third integrals, we note that the stationary points are given by

$$
f^{\prime}(k)=0
$$

where

$$
f(k)=\left\{\frac{k\left(1+D k^{4}\right)}{1+\epsilon k}\right\}^{1 / 2}-k \frac{x}{t} .
$$

It is easy to see that (4.1) has two positive roots when $x / t \geq 1$, but no root for $x / t<1$. Thus the stationary points are given by $k=\alpha_{1}$ and $k=\alpha_{2}$, where $\alpha_{1}$ and $\alpha_{2}$ are the only two positive real roots of

$$
\frac{1}{2}\left\{\frac{1+\epsilon k}{k\left(1+D k^{4}\right)}\right\}^{1 / 2} \frac{\left(1+5 D k^{4}+4 D \epsilon k^{5}\right)}{(1+k \epsilon)^{2}}-\frac{x}{t}=0 \quad\left(\frac{x}{t} \geq 1\right) .
$$


Thus we find that for large $x$ and $t$ such that $x / t \geq 1$,

$$
\eta(x, t) \approx \sum_{r=1}^{2} \frac{1}{2 \pi}\left(\frac{2 \pi}{t\left|f^{\prime \prime}\left(\alpha_{r}\right)\right|}\right)^{1 / 2} \cos \left(t f\left(\alpha_{r}\right) \pm \frac{\pi}{4}\right)
$$

where the sign \pm is to be chosen to agree with the sign of $f^{\prime \prime}\left(\alpha_{r}\right)$.

If the disturbance is in the form of an initial impulsive pressure $I(x)$ per unit area applied to the ice-covered surface, then only the initial conditions (2.11) and (2.12) are to be changed and are given, respectively, by

$$
\begin{gathered}
\phi-\epsilon \phi_{y}=-\frac{I(x)}{\rho} \quad \text { at } t=0, y=0, \\
\left(\phi-\epsilon \phi_{y}\right)_{t}=0 \quad \text { at } t=0, y=0,
\end{gathered}
$$

where $\rho=\bar{\rho} / l^{3}$.

If the initial impulse is concentrated at the origin, then by a similar analysis, we obtain for large $x$ and $t$ such that $x \geq t$,

$$
\eta(x, t) \approx-\frac{1}{2 \pi \rho} \sum_{r=1}^{2}\left(\left\{\frac{\alpha_{r}}{\left(1+\epsilon \alpha_{r}\right)\left(1+D \alpha_{r}^{4}\right)}\right\} \frac{2 \pi}{t\left|f^{\prime \prime}\left(\alpha_{r}\right)\right|}\right)^{1 / 2} \sin \left(t f\left(\alpha_{r}\right) \pm \frac{\pi}{4}\right)
$$

where the sign \pm is to be chosen to agree with the sign of $f^{\prime \prime}\left(\alpha_{r}\right)$ as before.

\section{Numerical results}

To study the effect of the presence of the ice cover on the wave motion generated due to initial disturbances at the upper surface, the asymptotic form of $\eta(x, t)$ is depicted graphically in Figures 5.1 and 5.2 for the case of an initial depression concentrated at the origin and in Figures 5.3 and 5.4 for the case of initial impulse also concentrated at the origin.

An obvious observation is the considerable reduction of the maximum amplitude of the generated waves due to the presence of the ice cover compared to the case of a free surface, and the oscillatory nature of the wave motion is quite prominent in the presence of ice cover. These figures show the variation of $\eta(x, t)$ at a fixed point $x$ when the time changes and at a fixed time $t$ when $x$ changes. The oscillatory nature of the wave motion in the presence of the ice cover compared to the case when there is no ice cover is more prominent in Figure 5.5.

Figures 5.6 and 5.7 depict the wave motion due to an initial depression of the ice cover against $x$ and $t$ (large) together, for two choices of values of $D$, namely, $D=0.5, D=0.1$, while Figures 5.8 and 5.9 depict the same due to initial impulsive pressure against $x$ and $t$ again for the same two choices of $D$.

Figures 5.10 and 5.11 depict the wave motion in the absence of the ice cover, against $x$ and $t$ (large). 


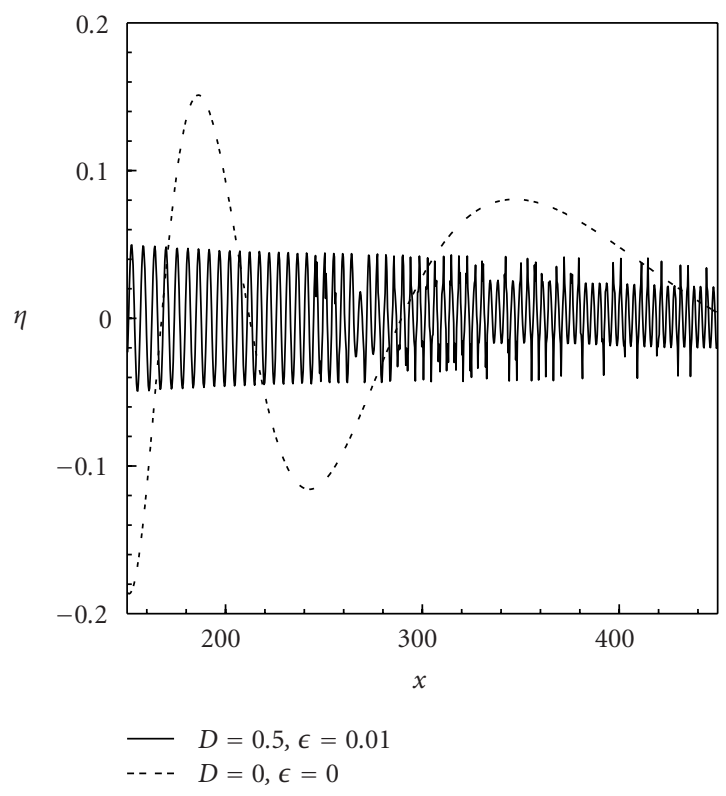

Figure 5.1. Wave motion due to initial displacement for fixed time $t=100 ; \epsilon=0.01, D=0.5$.

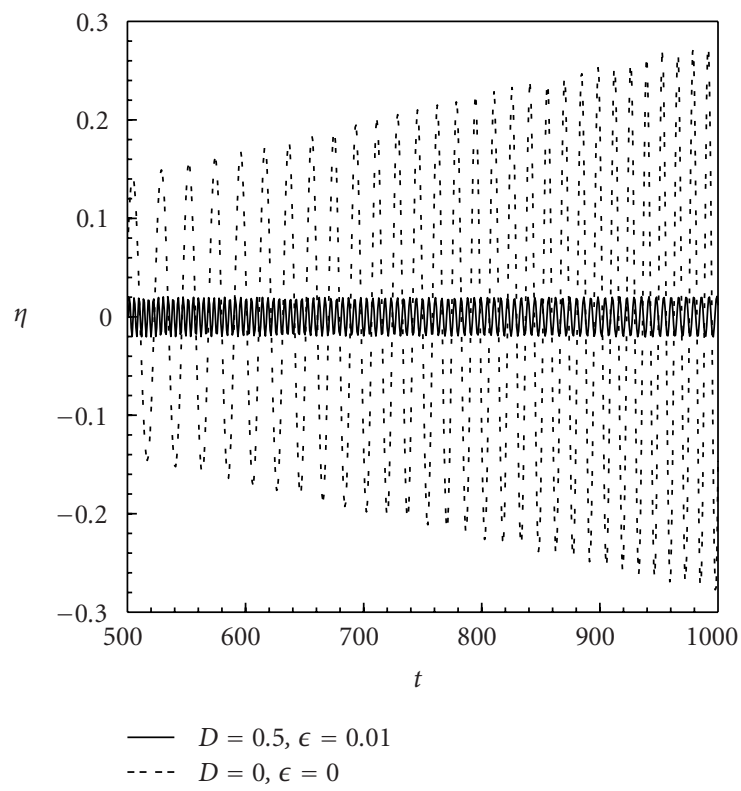

Figure 5.2. Wave motion due to initial displacement for fixed distance $x=1000 ; \epsilon=0.01, D=0.5$. 


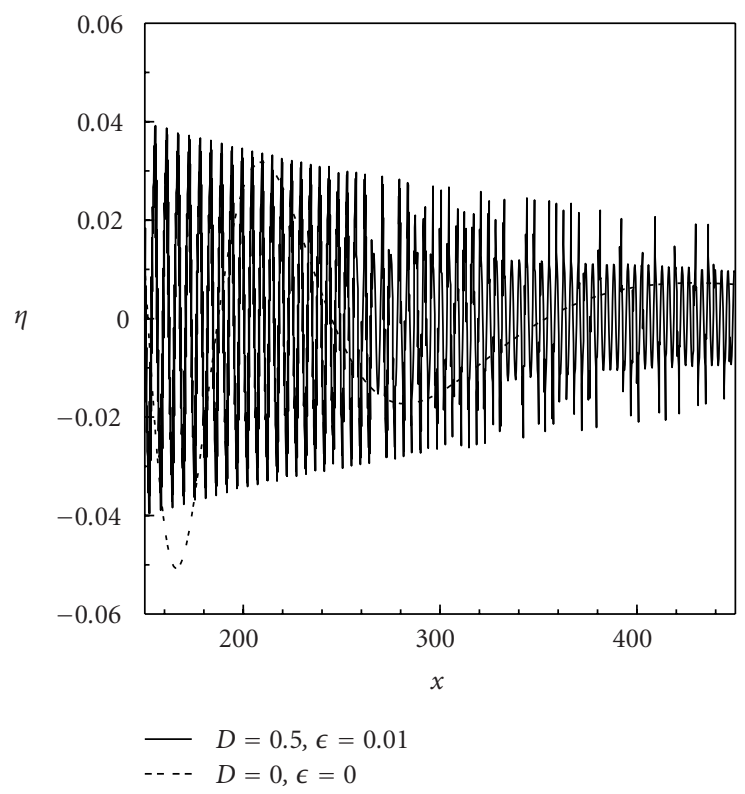

Figure 5.3. Wave motion due to impulse for fixed time $t=100 ; \epsilon=0.01, D=0.5$.

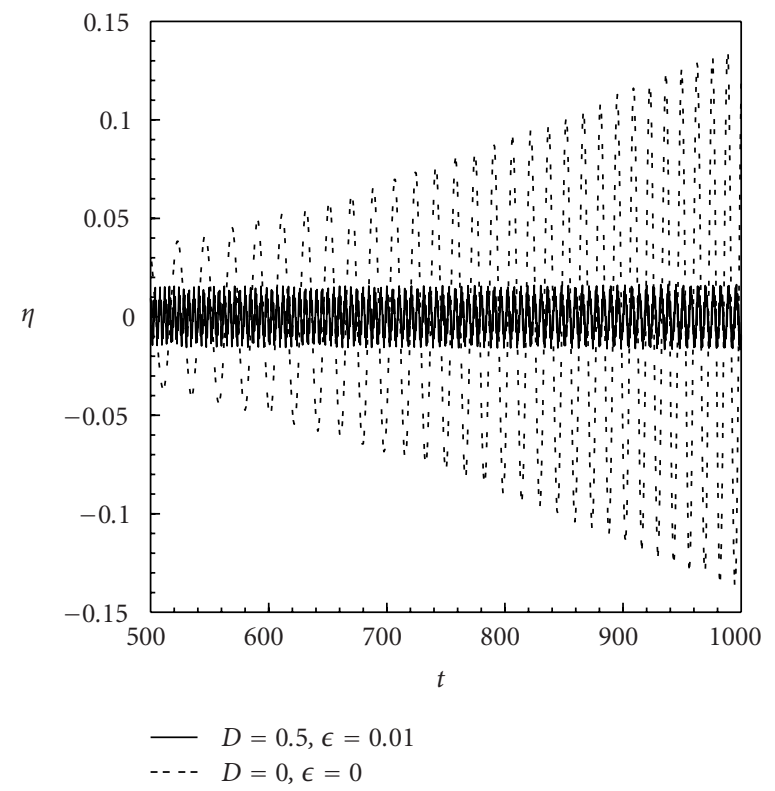

Figure 5.4. Wave motion due to impulse for fixed distance $x=1000 ; \epsilon=0.01, D=0.5$. 
Water waves generated by disturbances at an ice cover

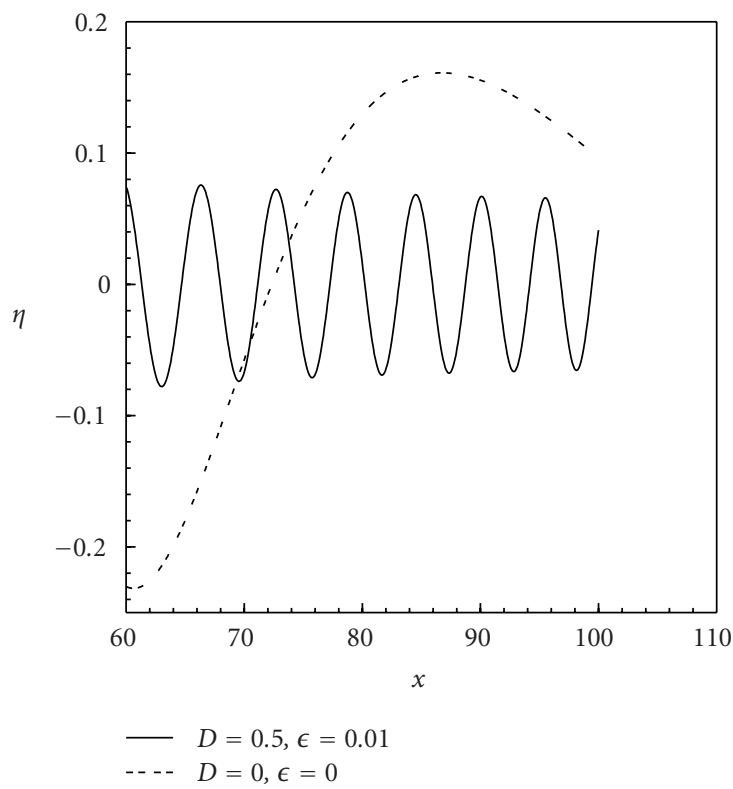

Figure 5.5. Wave motion due to initial displacement for fixed time $t=50 ; \epsilon=0.01, D=0.5$.

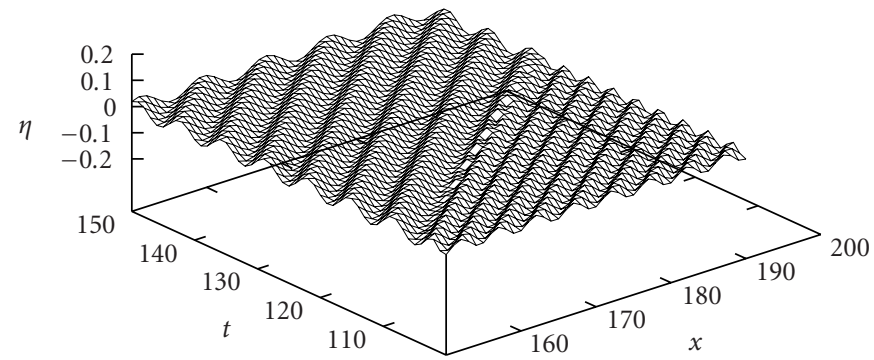

Figure 5.6. Wave motion due to initial displacement when $t=100$ to $150, x=150$ to $200 ; \epsilon=0.01$, $D=0.5$.

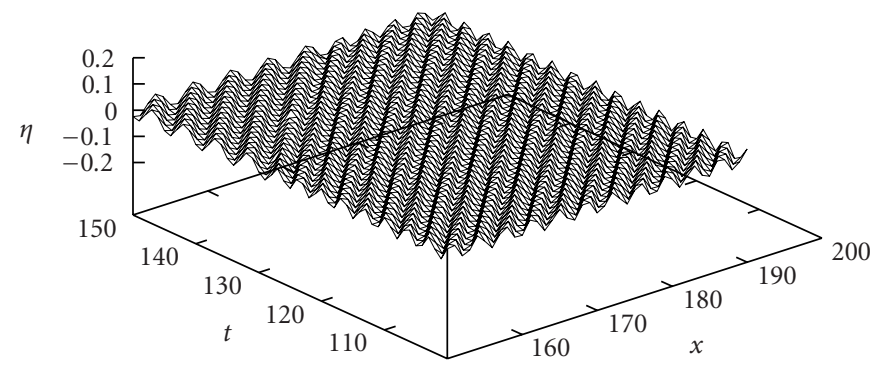

Figure 5.7. Wave motion due to initial displacement when $t=100$ to $150, x=150$ to $200 ; \epsilon=0.01$, $D=0.1$. 


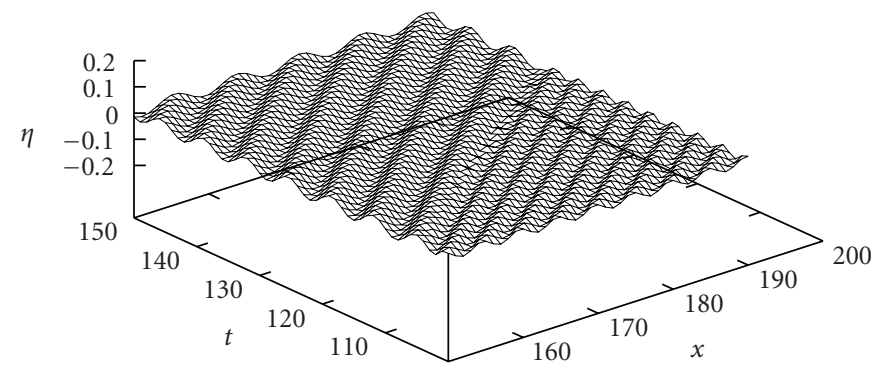

Figure 5.8. Wave motion due to impulse when $t=100$ to $150, x=150$ to $200 ; \epsilon=0.01, D=0.5$.

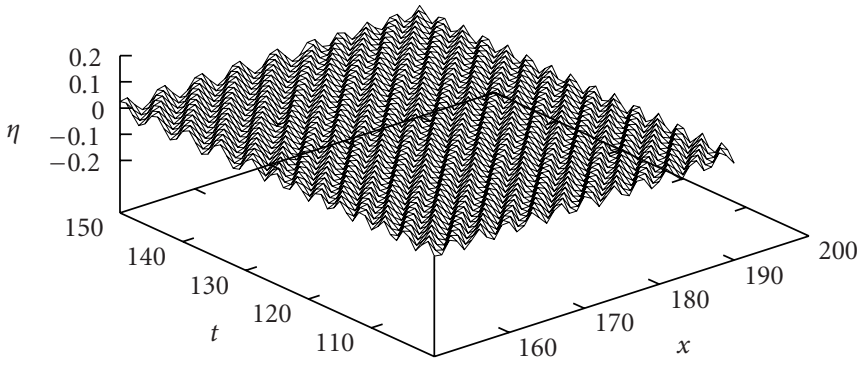

Figure 5.9. Wave motion due to impulse when $t=100$ to $150, x=150$ to $200 ; \epsilon=0.01, D=0.1$.

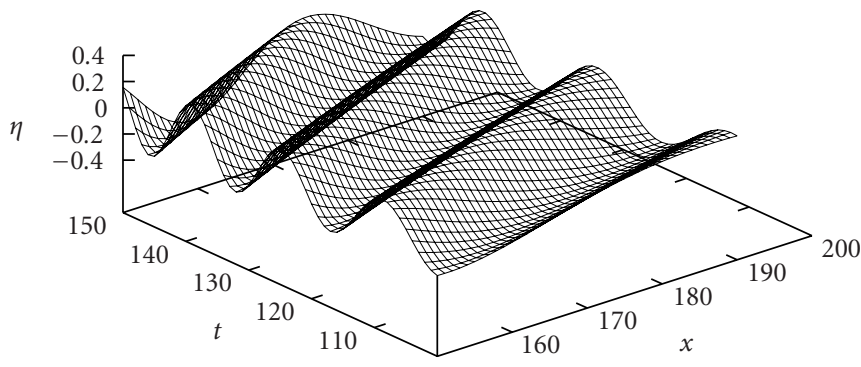

Figure 5.10. Wave motion due to initial displacement when $t=100$ to $150, x=150$ to $200 ; \epsilon=0$, $D=0$.

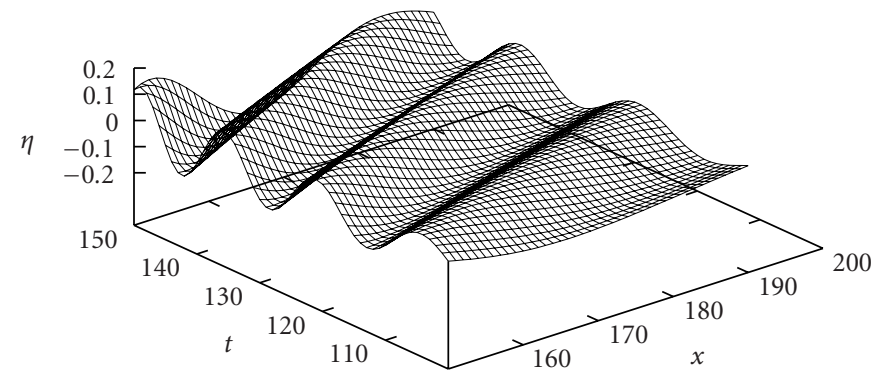

Figure 5.11. Wave motion due to impulse when $t=100$ to $150, x=150$ to $200 ; \epsilon=0, D=0$. 


\section{Water waves generated by disturbances at an ice cover}

These figures clearly show the effect of the presence of the ice cover on the wave motion. The presence of the ice cover reduces the amplitude of the wave motion but considerably increases the oscillatory nature. This may be attributed to the elastic behavior of the ice cover.

\section{Acknowledgment}

The authors thank the referees for their comments and suggestions.

\section{References}

[1] K. Chaudhury, Waves in shallow water due to arbitrary surface disturbances, Appl. Sci. Res. 19 (1968), 274-284.

[2] H. Chung and C. Fox, Calculation of wave-ice interaction using the Wiener-Hopf technique, New Zealand J. Math. 31 (2002), no. 1, 1-18.

[3] H. C. Kranzer and J. B. Keller, Water waves produced by explosions, J. Appl. Phys. 30 (1959), 398-407.

[4] H. Lamb, Hydrodynamics, Cambridge Mathematical Library, Cambridge University Press, Cambridge, 1993, with a foreword by R. A. Caflisch.

[5] B. N. Mandal, Water waves generated by disturbance at an inertial surface, Appl. Sci. Res. 45 (1988), no. 1, 67-73.

[6] J. J. Stoker, Water Waves: The Mathematical Theory with Applications, Pure and Applied Mathematics, Vol. IV, Interscience Publishers, New York, 1957.

Paramita Maiti: Physics and Applied Mathematics Unit, Physics and Earth Sciences Division, Indian Statistical Institute, 203 Barrackpore Trunk Road, Calcutta 700 108, India

E-mail address: pmaiti_r@isical.ac.in

B. N. Mandal: Physics and Applied Mathematics Unit, Physics and Earth Sciences Division, Indian Statistical Institute, 203 Barrackpore Trunk Road, Calcutta 700 108, India

E-mail address: biren@isical.ac.in 


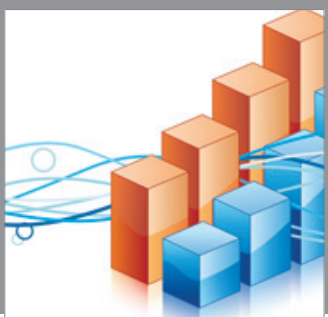

Advances in

Operations Research

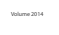

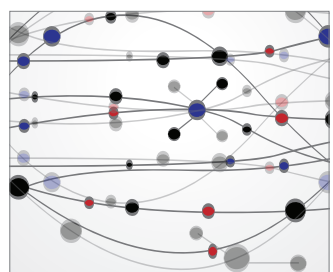

\section{The Scientific} World Journal
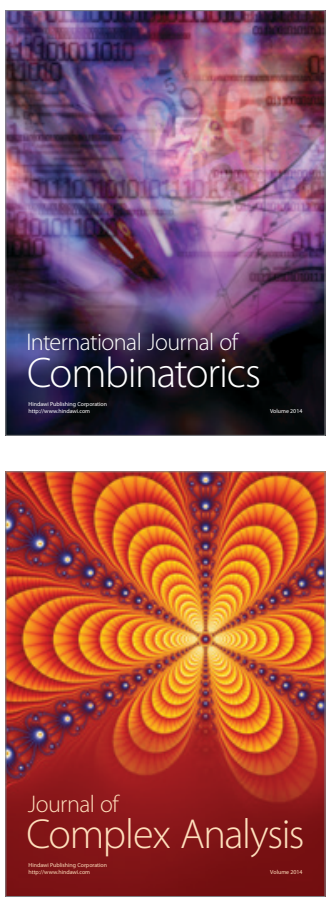

International Journal of

Mathematics and

Mathematical

Sciences
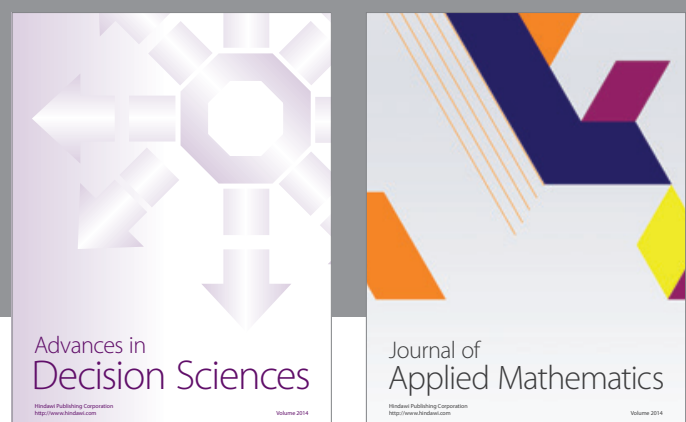

Journal of

Applied Mathematics
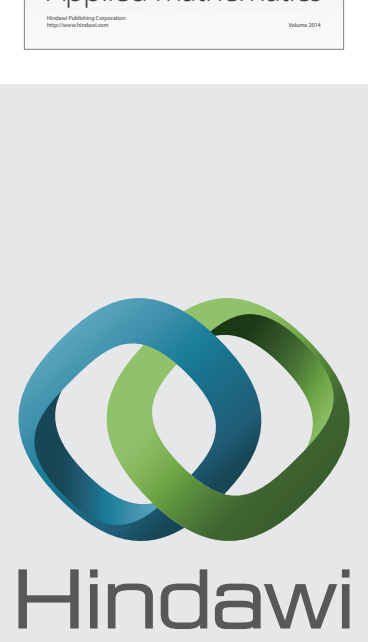

Submit your manuscripts at http://www.hindawi.com
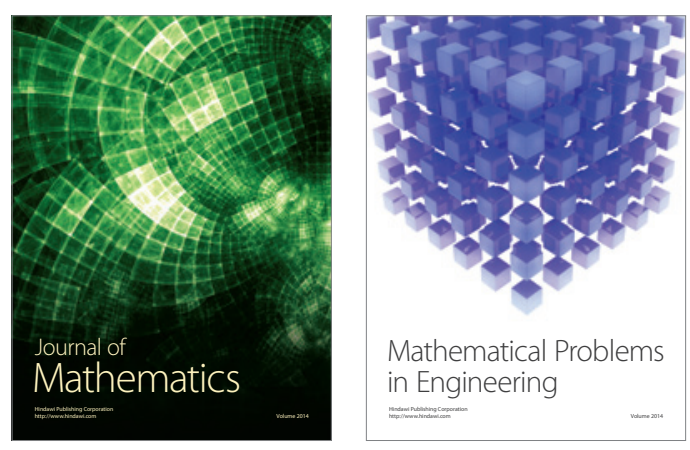

Mathematical Problems in Engineering
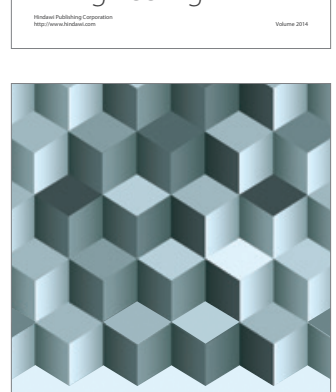

Journal of

Function Spaces
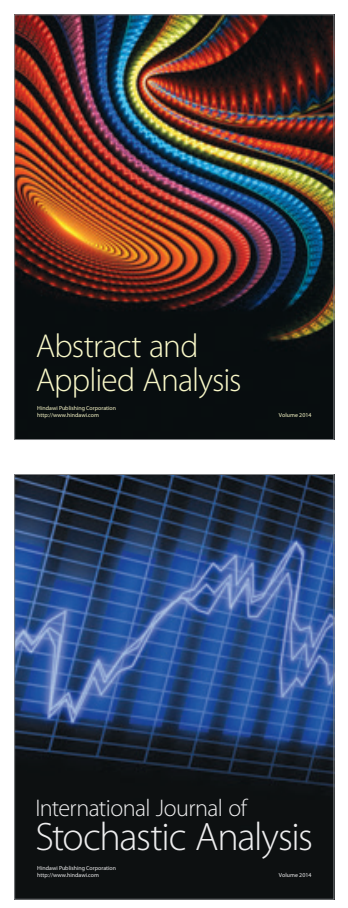

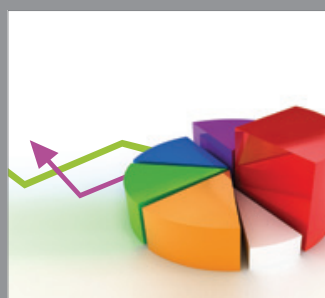

ournal of

Probability and Statistics

Promensencen
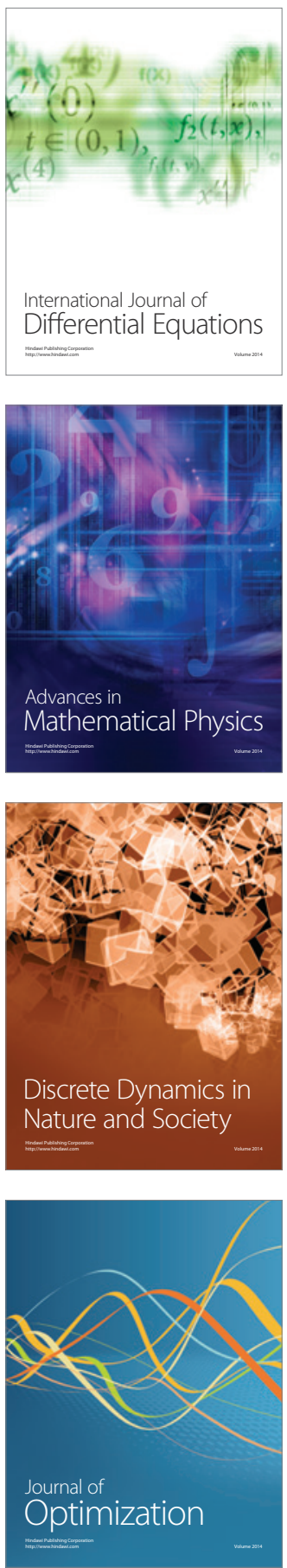